\title{
The Value and Sustainability of Ocrelizumab in Relapsing Multiple Sclerosis: A Cost-Effectiveness and Budget Impact Analysis
}

\author{
Paolo Angelo Cortesi ${ }^{1}$, Damiano Paolicelli ${ }^{2}$, Marco Capobianco ${ }^{3}$, Paolo Cozzolino ${ }^{1}$, \\ Lorenzo Giovanni Mantovani ${ }^{1}$ \\ Research Centre on Public Health (CESP), University of Milano-Bicocca, Monza, Italy \\ Department of Basic Medical Sciences, Neuroscience and Sense Organs, University of Bari" Aldo Moro", Bari, Italy \\ Department of Neurology, Regional Multiple Sclerosis Centre (CReSM), University-hospital San Luigi Gonzaga, Orbassano (TO), Italy
}

\begin{abstract}
INTRODUCTION: The availability of ocrelizumab for the relapsing forms of multiple sclerosis (MS) in the Italian markets raised some questions about its economic impact and value compared to the alternative treatment options available.

AIM: To assess the cost-effectiveness and budget impact of ocrelizumab compared to the most used second line disease modifying therapies (DMTs) in Italy.

METHODS: The study was divided in two phases: Phase 1 - based on the development of a decision analytical Markov model to assess the cost-effectiveness of ocrelizumab compared to natalizumab and fingolimod, and Phase 2 - based on the development of a budget impact model to assess the economic impact of ocrelizumab in Italy. Both models used the National Health System perspective; a lifetime horizon was applied in the cost-effectiveness analysis and a 3-year time horizon in the budget impact. The cost-effectiveness analysis results were reported as incremental cost-effectiveness ratio (ICER) expressed as $€$ per Quality Adjusted Life Year (QALY) gained, the budget impact analysis results were reported as difference in the overall budget $(€)$ between a scenario with and without ocrelizumab.

RESULTS: The two analyses reported ocrelizumab as a cost-effective option compared to natalizumab and fingolimod with a positive impact on the overall NHS budget. In the base-case analysis, the ICER was $€ 2,023$ for ocrelizumab compared to fingolimod; while ocrelizumab resulted cost-saving compared to natalizumab. The sensitivity analysis confirmed the base-case analysis results. Further, the use of ocrelizumab was associated to a budget decrease of $€ 21$ million $(-2.6 \%)$ in a 3-year time horizon.

CONCLUSION: The results of our cost-effectiveness and budget impact models reported ocrelizumab as an effective and efficient treatment in patients with relapsing forms of MS who failed a first line DMTs from the Italian NHS perspective.
\end{abstract}

\section{Keywords}

Disease Modifying Therapies; Relapsing Multiple Sclerosis; Cost-effectiveness

\section{BACKGROUND}

Multiple sclerosis (MS) is the most prevalent chronic inflammatory disease of the central nervous system, affecting more than 2 million people worldwide [1] and remains the major cause of neurological disability in young adults [1,3]. MS often leads to significant accumulated disability over its typical 30-40-year course although the course is highly variable.

MS is associated to a high economic burden, with an estimated annual cost of approximately $\$ 10$ billion in USA $[4,5]$. MS reported high direct costs associated to the health care resources consumption, but also high indirect costs associated to informal care, services and loss of productivity [5]. The direct and indirect costs are associated to the disability status of MS patients, with increased costs associated to increased disability [5]. The introduction of disease-modifying therapies (DMTs) over the past two decades has had profound effects on the management of Relapsing Multiple Sclerosis (RMS), reducing the progression of the disease and changing the cost drivers in the low level of disability [5-7]. However, there is still need of new effective and safe treatment to improve the management of RMS and avoiding the development of high disability level [7].

Corresponding author Paolo Angelo Cortesi paolo.cortesi@unimib.it Published: 10 July 2019 
In this scenario, ocrelizumab is a humanized anti-CD20 monoclonal antibody recently approved in Italy for the treatment of adults with RMS and with primary progressive form (PPMS). In the 96-week OPERA I and OPERA II trials, patients with RMS and treated with ocrelizumab significantly reduced annualized relapse rates and disability progression versus interferon $\beta$-1a [8]. The availability of this new DMT in the market raised some questions about its value compared to the alternative treatment options and its economic impact. To fill this gap, we aimed to assess the cost-effectiveness and budget impact of ocrelizumab compared to most used second line treatment options available in Italy.

\section{METHODS}

The study was divided in two phases: Phase 1 - based on the development of a decision analytical Markov model to assess the cost-effectiveness of ocrelizumab, and Phase 2 - based on a budget impact model to assess the economic impact associated to the market access of ocrelizumab in Italy.

\section{Phase 1: Cost-Effectiveness Analysis}

The cost-effectiveness analysis was based on a Markov model developed to assess ocrelizumab in patients with RMS who failed a first line treatment. The model simulated the natural history of RMS based on four main clinical events: disability progression, relapse, development of progressive form, and death. The Expanded Disability Status Scale (EDSS) score defined the level of disability and its progression. The model included the impact of ocrelizumab and other DMTs applying a reduction in the disability progression and relapse rate with positive effects on the patient's health and quality of life. The model also simulated the cost associated to the RMS management and the cost associated to DMTs.

Figure 1 reports the model structure. The health states were defined based on the EDSS score, with each level of EDSS representing one health state. This approach was in line with previous models developed to assess DMTs in RMS [9]. At the beginning of the simulation, the RMS patients reported an EDSS level within 0 and 6.5. During the simulation, these patients could develop a higher or lower EDSS level or the Secondary Progressive Multiple Sclerosis (SPMS) course. When simulated patients developed SPMS, the model not allows moving back to the RMS. Further, as done for the RMS, the SPMS severity was defined by the EDSS level [9]. The SPMS patients could experience an EDSS level between 2 and 9 and

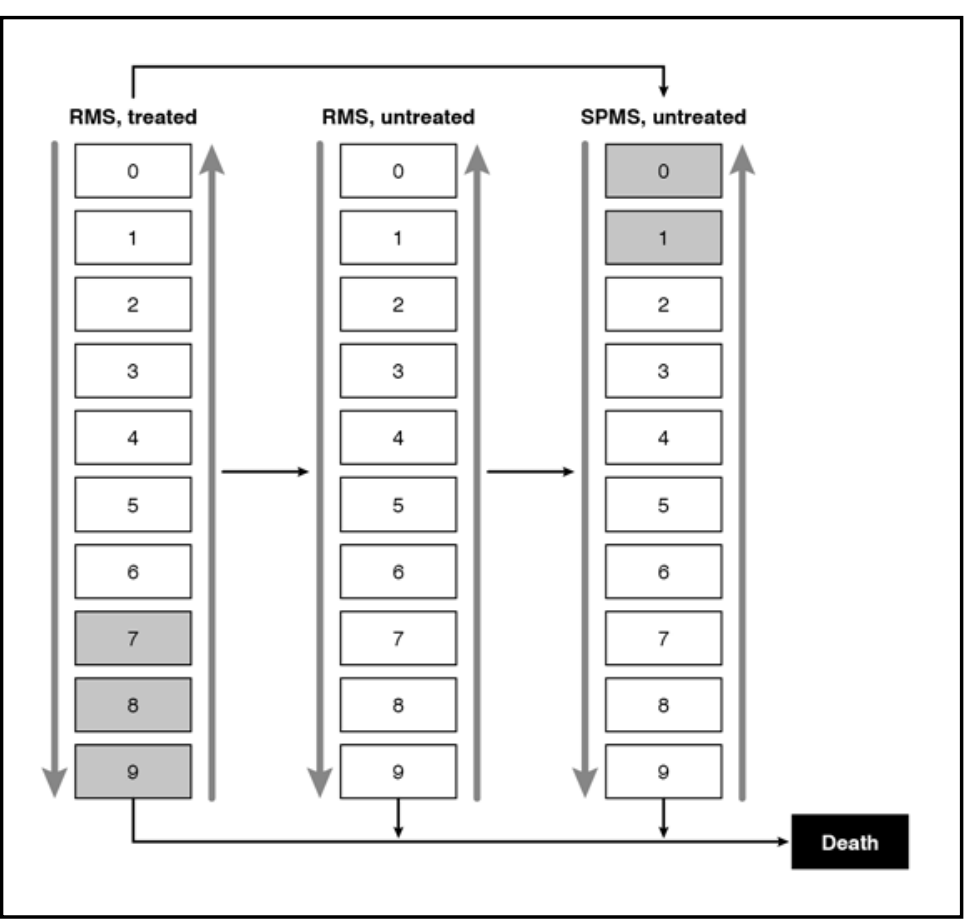

Figure 1. Cost-effectiveness model structure. Grey arrows show the possible direction of EDSS level changes during the simulation; Grey squares in the "RMS, treated" health states represent the EDSS leve/ where the diseases modifying treatments are not provided; Grey squares in the "SPMS, untreated" health states represent the EDSS level not allow in the simulation for the SPMS type RMS = relapsing remitting multiple sclerosis; SPMS = secondary progressive multiple sclerosis. move to higher EDSS level during the simulation. All the DMTs included in the model could reduce the disability progression and the relapse rate in RMS patients. Further, the model assumes an effect of DMTs in reducing the development of SPMS. The DMTs interruption was assumed when patients developed an EDSS level equal or higher than seven or when they developed SPMS.

In the model, the disability progression probability and relapse rate were kept constant over time and were specific for the MS phenotype (RMS or SPMS) and EDSS level. The mortality rates applied in the model were specific for age, sex and EDSS level. No direct effect on mortality was associated to DMTs, but an indirect effect was assumed based on the treatment effect in reducing disease progression to higher EDSS level, that were associate to a higher probability of death.

In this cost-effectiveness analysis, the model compared ocrelizumab (Ocrevus ${ }^{\circledR}$, Roche S.p.A.) with the most frequent used second line DMTs: fingolimod (Gilenya ${ }^{\circledR}$, Novartis Farma S.p.A.) and natalizumab (Tysabri $^{\circledR}$, Biogen Italia s.r.1.). The model estimated costs and benefits of each treatment 
and expressed outcomes in terms of 2019 euro $(€)$, life-years (LYs), quality-adjusted lifeyears (QALYs), and incremental cost-effectiveness ratios (ICERs) expressed as $€$ per QALY gained.

We discounted costs and benefits at an annual $3 \%$ rate $[10,11]$ and performed the analysis from an Italian National Health System (NHS) perspective. The model adopted a lifetime horizon and used a 1-year cycle length for the simulation.

\section{Clinical Data}

The simulated cohort in the cost-effectiveness analysis reflected the mean age, gender and EDSS distribution reported in the OPERA I and OPERA II trials [8] (Table I).

The probabilities of EDSS changes in RMS were estimated from the British Columbia Multiple Sclerosis database [20]. Estimates of the probability of moving from RMS to SPMS for each EDSS level and the probability of the transition among EDSS level in SPMS patients derived from the London Ontario database [12-16] (Table I). A specific relapse probability for each EDSS level was estimated for both RMS and SPMS and were estimated combining the data by Patzold et al. [15] with data reported in the UK MS Survey [17] (Table I).

The mortality probabilities associated to MS were based on the high mortality rates reported by MS patients compared to the general population $[18,19]$. The specific Italian general population mortality rates, stratified by age and sex [23], were adjusted by mortality relative risk reported by Pokorski et al. [19] for each EDSS level (Table I). These relative risks were estimated comparing the mortality risk of MS in each EDSS level with the mortality rate in the general population, adjusting for age and sex [19].

\section{Treatment Effectiveness}

In this cost-effectiveness analysis, ocrelizumab was compared with the most used second line DMTs in Italy (fingolimod and natalizumab). These treatments modify the natural history of RMS i) slowing down the disease progression to higher EDSS level, ii) reducing the relapse rate, and iii) reducing the progression to SPMS.

A recently published Network Meta-Analysis (NMA) [24], based on DMTs clinical trials, reported the relative risk (RR) of annual relapse rate of each treatment compared to placebo (Table II). Further, using the placebo as reference, the NMA estimated the efficacy of each treatment in reducing the disability progression. The treatment efficacy was reported as Hazard ratio (HR) using the Confirmed Disability Progression (CDP) at 12 weeks (Table II). To estimate the reduction of relapse rate and disease progression associated to each treatment, we applied the RR and HR estimated in the NMA to the natural history probabilities.

\begin{tabular}{|c|c|c|c|c|c|c|c|c|c|c|c|}
\hline \multirow{2}{*}{$\begin{array}{c}\text { Parameters } \\
\text { EDSS }\end{array}$} & \multicolumn{10}{|c|}{ Value } & \multirow{2}{*}{ Source } \\
\hline & 0 & 1 & 2 & 3 & 4 & 5 & 6 & 7 & 8 & 9 & \\
\hline \multicolumn{12}{|l|}{ Cohort characteristics } \\
\hline Age (years) & \multicolumn{10}{|c|}{37.0} & [8] \\
\hline Male (\%) & \multicolumn{10}{|c|}{34.0} & [8] \\
\hline Disability distribution (\%) & 3.08 & 18.85 & 30.45 & 23.50 & 14.74 & 8.76 & 0.60 & - & - & - & [8] \\
\hline \multicolumn{12}{|c|}{ Clinical data (annual probability) } \\
\hline Developing SPMS & 0.000 & 0.045 & 0.074 & 0.094 & 0.119 & 0.151 & 0.189 & 0.237 & 0.295 & 1.000 & {$[12-16]$} \\
\hline Relapse-RMS & 0.710 & 0.730 & 0.680 & 0.720 & 0.710 & 0.590 & 0.490 & 0.510 & 0.510 & 0.510 & {$[15,17]$} \\
\hline Relapse - SPMS & - & - & 0.470 & 0.880 & 0.550 & 0.520 & 0.450 & 0.340 & 0.340 & 0.340 & {$[15,17]$} \\
\hline $\begin{array}{l}\text { Mortality (RR) -RMS and } \\
\text { SPMS }\end{array}$ & 1.000 & 1.430 & 1.600 & 1.640 & 1.670 & 1.840 & 2.270 & 3.100 & 4.450 & 6.450 & {$[18,19]$} \\
\hline \multicolumn{12}{|l|}{ Quality of life (utility) } \\
\hline RMS & 0.923 & 0.882 & 0.836 & 0.777 & 0.783 & 0.755 & 0.718 & 0.579 & 0.310 & 0.040 & {$[21]$} \\
\hline SPMS & 0.878 & 0.837 & 0.791 & 0.732 & 0.738 & 0.710 & 0.673 & 0.534 & 0.265 & -0.005 & {$[5,21]$} \\
\hline \multicolumn{12}{|c|}{ Costs (overall management) } \\
\hline RMS (€) & 1,906 & 1,906 & 1,906 & 1,906 & 3,983 & 3,983 & 3,983 & 9,468 & 9,468 & 9,468 & [22] \\
\hline SPMS (€) & 5,347 & 5,347 & 5,347 & 5,347 & 9,642 & 9,642 & 9,642 & 9,517 & 9,517 & 9,517 & [22] \\
\hline
\end{tabular}

Table I. Clinical, quality of life and costs data input

EDSS = the Expanded Disability Status Scale; RMS = Relapsing Multiple Sclerosis; RR = relative risk; SPMS = Secondary Progressive Multiple Sclerosis 


\begin{tabular}{lcccc|ccc}
\hline \multirow{2}{*}{ DMT } & \multicolumn{2}{c}{ ARR } & \multicolumn{2}{c|}{ CDP-12 weeks } & \multicolumn{2}{c}{ Discontinuation } \\
\cline { 2 - 8 } & RR & SE & HR & SE & Annual probability & SE \\
\hline Fingolimod & 0.472 & 0.078 & 0.777 & 0.119 & 0.063 & 0.006 \\
Natalizumab & 0.376 & 0.105 & 0.536 & 0.176 & 0.022 & 0.002 \\
Ocrelizumab & 0.306 & 0.125 & 0.432 & 0.235 & 0.062 & 0.006 \\
\hline
\end{tabular}

Table II. Disease-modifying therapies (DMTS) efficacy and discontinuation probability [24]

$\mathrm{ARR}=$ annualized relapse rate; $\mathrm{CDP}=$ confirmed disability progression; HR, Hazard ratio; $\mathrm{RR}$ = relative risk; $\mathrm{SE}=$ standard error

Using a conservative approach, considering the efficacy of ocrelizumab in the MS progressive phenotypes, we assumed no treatments efficacy in SPMS. Further, we assumed the probability of treatment interruption for any causes reported by the NMA [24]. This approach gave the possibility to account for both adverse events (AEs) and reduction of efficacy (Table II). The simulated patients moved to the "not treated status" when they discontinued the treatment. The probabilities of main adverse events for each treatment were retrieved form the NMA (see Supplementary Files, Table I) [24]. Finally, we also included the most important DMTs AEs based on the clinical experts' opinion.

\section{Quality of Life}

Specific Italian utility values for each EDSS level derived from Battaglia et al. [21]; while the disutility associates to a relapse event was retrieved by Kobelt et al. [5] and assumed equal to -0.18 in all EDSS levels. The relapse disutility was estimated as a difference between patients who experienced a relapse and patients who do not reported any relapse in the survey conducted by Kobelt et al. The model assumed relapse duration of 46 days.

The utility for each EDSS level in the SPMS patients was estimated subtracting 0.045 to the RMS utility values (Table I). The utility reduction was estimated from the date reported in Orme et al. [17]. Further, the AEs disutility was estimated based on the health care resources consumption reported in two NICE appraisals on MS treatment (see Supplementary Files, Table I) $[25,26]$.

\section{Cost Data}

The cost-effectiveness analysis was conducted from the Italian NHS perspective, therefore, we included the costs associated to the overall disease management for each EDSS level, the DMTs treatment cost (drug costs + treatment follow-up costs), and the cost due to relapses.

The MS management cost was retrieved by an Italian cost-of-illness study [22]. Table I reports the cost for each EDSS level, excluding the DMTs costs that are accounted in the model as a specific and independent parameter. The costs were adjusted for the inflation rate reported in Italy and estimated as $€$ in 2018 [27].

As already reported, the management costs were estimated for each EDSS level, excluding the costs associated to the DMTs, and include hospitalizations, pharmacological therapies, outpatient visits, and diagnostic exams not associated to DMTs. The DMTs costs were considered only in RMS patients with EDSS $<7$ in the treatment status and include drug costs, administration costs, and outpatient visits, laboratory and diagnostic examinations associated to the treatment follow-up.

The DMT cost was estimated as annual treatment cost, based on ex-factory price [28] (Table III). The final DMTs cost was estimated including the statutory and hidden discounts.

The administration cost of injectable DMTs was $€ 11.6$ per administration [29]. The annual treatment follow-up costs were estimated for each DMT based on the health care resources consumption reported in the Emilia Romagna Region guidelines and on the national tariff reported by AGENAS [29-31]. The relapse cost was retrieved by an Italian cost-of-illness study and set to $€ 405$ per relapse [22]. Further, the AEs costs were estimated based on the health care resources consumption reported in two NICE appraisals on MS treatments (see Supplementary Files, Table I). [25,26].

\section{Outcome}

The model estimated the costs $(€)$, the life years and the QALYs gained for each DMT included in the analysis. The cost-effectiveness results were reported as incremental costeffectiveness ratio (ICER) per QALY gained. The ICER was estimated comparing ocrelizumab with natalizumab and fingolimod. An ICER under the willingness-to-pay threshold of $40,000 €$ per QALY gained was considered cost-effective [37]. 


\begin{tabular}{|c|c|c|c|c|c|c|c|c|}
\hline DMT & Dosage & Package & $\begin{array}{c}\text { List } \\
\text { price }(€)\end{array}$ & $\begin{array}{c}\text { Net } \\
\text { price }(€)\end{array}$ & Source & $\begin{array}{l}\text { Follow-up } \\
\text { 1st year } \\
(€)\end{array}$ & $\begin{array}{l}\text { Follow-up } \\
\text { after } 1 \text { st } \\
\text { year }(€)\end{array}$ & Source \\
\hline Fingolimod & 0.5 mg qd, PO & 28 capsules 0.5 mg & 2,681 & 1,624 & [28] & 1,203 & 309 & [29-31] \\
\hline Natalizumab & $\begin{array}{l}300 \text { mg every } \\
4 \text { weeks, IV }\end{array}$ & $300 \mathrm{mg} / 15 \mathrm{ml}, 1$ unit & 2,681 & 1,624 & [28] & 1,737 & 937 & \\
\hline Ocrelizumab & $\begin{array}{l}600 \text { mg every } \\
6 \text { months, IV }\end{array}$ & $300 \mathrm{mg} / 10 \mathrm{ml}, 1$ unit & 9,309 & 5,641 & [28] & 1,150 & 363 & \\
\hline Alemtuzumab & $\begin{array}{l}12 \mathrm{mg} / \text { day for } 5 \text { days } \\
\text { (first cycle); after } 12 \\
\text { months, } 12 \mathrm{mg} / \text { day } \\
\text { for } 3 \text { days (second } \\
\text { cycle), IV }\end{array}$ & $12 \mathrm{mg} / 1.2 \mathrm{ml}, 1$ vial & 13,126 & 7,953 & [28] & 2,105 & 1,233 & \\
\hline
\end{tabular}

Table III. Disease-modifying therapies (DMTs) costs

IV = intravenous administration; $\mathrm{PO}=$ per os (oral administration); qd = quaque die (once a day)

\section{Sensitivity analyses}

A series of sensitivity analyses were performed to assess the reliability of model results. An alternative scenario was assessed using the CDP-24 weeks instead of CDP-12 based on the data reported in the NMA [24]. A second alternative scenario was assessed using the society's perspective to include the possible impact of ocrelizumab on the direct non-medical costs and the indirect costs compare to the other DMTs included. These costs were retrieved by Battaglia et al. for each EDSS level [21] (see Supplementary Files, Table II). Further, a one-way sensitivity analysis was performed to assess the impact of each parameter on the model results and a probabilistic sensitivity analysis was performed to assess the impact of all parameters uncertainty.

\begin{tabular}{lcc}
\hline \multicolumn{1}{c}{ Parameter } & Value & Source \\
\hline Italian population (n.) & $60,665,551$ & {$[35]$} \\
MS prevalence & 143 per 100,000 population & {$[32,33]$} \\
MS incidence & 5.7 per 100,000 person-years & {$[32]$} \\
MS mortality & 0.49 per 100 person-years & {$[33,34]$} \\
$\begin{array}{l}\text { RMS prevalence within MS } \\
\text { patients (\%) }\end{array}$ & 79.3 & {$[36]$} \\
$\begin{array}{l}\text { Rapidly evolving severe RMS } \\
\text { prevalence, within RMS (\%) }\end{array}$ & 10.0 & {$[36]$} \\
$\begin{array}{l}\text { Patients treated with DMTs (\%) } \\
\begin{array}{l}\text { Patients treated with } \\
\text { alemtuzumab, fingolimod and } \\
\text { natalizumab, within treated } \\
\text { patients (\%) }\end{array}\end{array}$ & 63.9 & {$[36]$} \\
\hline
\end{tabular}

Table IV. Target population of the budget impact analysis DMTs = Disease-modifying therapies; MS = multiple sclerosis; RMS = relapsing multiple sclerosis

\section{Phase 2: Budget Impact Analysis}

The analysis was based on a Budget Impact model developed to assess the economic impact of ocrelizumab on the RMS management in the Italian market. The model estimated the costs associated to MS management in a scenario where ocrelizumab was not available (Scenario No-Ocre) compared to a scenario where ocrelizumab was available (Scenario Ocre).

The model was based on epidemiological data of RMS in Italy and on the second-line DMTs market share. The model estimated the RMS patients in Italy treated with a second line DMTs (alemtuzumab, fingolimod and natalizumab) or with a rapidly evolving severe RMS. The model estimated the number of patients treated with each DMT and the associated costs using 3-year time horizon and from the Italian NHS perspective. Based on the NHS point of view, the model included the cost of DMTs, follow-up (outpatient visits, laboratory tests, and diagnostic exams) and AEs management.

\section{Population}

The model estimated the Italian MS population using an average prevalence of $141.7 \mathrm{per}$ 100,000 population [32,33], a yearly incidence of 5.7 per 100,000 person-years [32] and a mortality rate of 0.49 per 100,000 person-years [33,34] (Table IV). Based on the MS population, the model estimates the number of RMS patients and the number of patients with rapidly evolving severe RMS (Table IV). The rapidly evolving patients and the RMS patients treated with alemtuzumab, fingolimod and natalizumab were combined to estimate the target population of ocrelizumab budget impact analysis (Table IV). In the 3 years simulated, we assumed no change in the incidence and mortality rate.

\section{Costs}

Considering the Italian NHS perspective, we included the following cost: i) DMTs cost, ii) monitoring and follow-up costs, and iii) AEs costs. 


\begin{tabular}{lcc|cc|cc}
\hline \multicolumn{1}{c}{ DMT } & \multicolumn{2}{c}{ Year 1 } & \multicolumn{2}{c}{ Year 2 } & \multicolumn{2}{c}{ Year 3 } \\
\cline { 2 - 7 } & $\begin{array}{l}\text { Scenario w/o } \\
\text { ocrelizumab }\end{array}$ & $\begin{array}{l}\text { Scenario w/ } \\
\text { ocrelizumab }\end{array}$ & $\begin{array}{c}\text { Scenario w/o } \\
\text { ocrelizumab }\end{array}$ & $\begin{array}{l}\text { Scenario w/ } \\
\text { ocrelizumab }\end{array}$ & $\begin{array}{c}\text { Scenario w/o } \\
\text { ocrelizumab }\end{array}$ & $\begin{array}{c}\text { Scenario w/ } \\
\text { ocrelizumab }\end{array}$ \\
\hline Ocrelizumab & - & 15 & - & 30 & - & 45 \\
Fingolimod & 60 & 50 & 60 & 45 & 60 & 37,5 \\
Natalizumab & 35 & 30 & 35 & 20 & 35 & 12,5 \\
Alemtuzumab & 5 & 5 & 5 & 5 & 5 & 5 \\
\hline
\end{tabular}

Table V. Disease-modifying therapies (DMT) market share in the 3 simulated years

The cost associated to relapses and EDSS level were not included in the budget impact analysis due to the low cost of relapse [22] and to the short time horizon assumed for this analysis that not allowed observing a significant impact in the EDSS progression of the different simulated treatments.

The DMTs costs are reported in Table III. The monitoring and follow-up costs used in the budget impact model are the same described for the cost-effectiveness analysis (Table III).

Further, also the AEs costs are the same used in the cost-effectiveness analysis [24-26] (see Supplementary Files, Table I).

\section{Market share}

The market share in the Scenario No-Ocre was estimated based on the DMTs utilization in Italy and reported in the Barometro 2017 report [32] in which, alemtuzumab, fingolimod and natalizumab were the $24.3 \%$ of all DMTs used in Italy (Table IV). Of these three DMTs, fingolimod was the most used and alemtuzumab the less frequent one (Table V). This market distribution was assumed constant over the 3 years simulation.

In the Scenario-Ocre, we assumed a $15 \%$ use of ocrelizumab in the first year, $30 \%$ in the second year and $45 \%$ in the third year (Table V). The increasing use of ocrelizumab was balanced by the reduction of natalizumab and fingolimod. No change in the alemtuzumab market share was assumed in the Scenario-Ocre over the 3 years simulated.

In the analysis, we assumed a constant rate of treatment switch over each simulated year. Further, in the model we assumed a yearly treatment discontinuation probability of $12.1 \%$ [22].

\section{Outcome}

Based on the costs and health care resources consumption, the model estimated the total annual cost per patient per each treatment. The estimated annual costs were combined with

\begin{tabular}{|c|c|c|c|c|c|c|c|}
\hline DMT & Costs (€) & $\Delta$ Costs $(€)$ & LYs & $\Delta$ LYs & QALYs & $\triangle$ QALYs & $\begin{array}{l}\text { ICER }^{1} \text { ( } € \text { per } \\
\text { QALY gained) }\end{array}$ \\
\hline \multicolumn{8}{|c|}{ Base-case scenario } \\
\hline Ocrelizumab & 253,842 & - & 21.704 & - & 12.706 & - & - \\
\hline Natalizumab & 306,798 & $-52,956$ & 21.628 & 0.076 & 12.200 & 0.506 & Dominant \\
\hline Fingolimod & 251,197 & 2,645 & 21.423 & 0.281 & 11.399 & 1.308 & 2,023 \\
\hline \multicolumn{8}{|c|}{ Alternative scenario 1 - CDP-24 weeks as effectiveness outcome } \\
\hline Ocrelizumab & 252,143 & - & 21.642 & - & 12.432 & - & - \\
\hline Natalizumab & 317,173 & $-65,030$ & 21.783 & -0.140 & 12.820 & -0.386 & $168,421^{2}$ \\
\hline Fingolimod & 252,658 & -515 & 21.462 & 0.181 & 11.588 & 0.846 & Dominant \\
\hline \multicolumn{8}{|c|}{ Alternative scenario 2 - Societal perspective } \\
\hline Ocrelizumab & 827,194 & - & 21.704 & - & 12.706 & - & - \\
\hline Natalizumab & 902,324 & $-75,147$ & 21.628 & 0.076 & 12.200 & 0.506 & Dominant \\
\hline Fingolimod & 887,203 & $-60,009$ & 21.423 & 0.281 & 11.399 & 1.308 & Dominant \\
\hline
\end{tabular}

Table VI. Cost-effectiveness analysis results

$\mathrm{CDP}=$ confirmed disability progression; Dominant = ocrelizumab more effective and less expensive; ICER = incremental cost-effectiveness ratio; LYs = life years; QALYS = Quality Adjusted Life Years

${ }^{1}$ ICER estimated comparing ocrelizumab vs other DMTs

${ }^{2}$ ICER estimated comparing natalizumab vs ocrelizumab due to the high cost and effectiveness reported by natalizumab in the Alternative scenario 1 


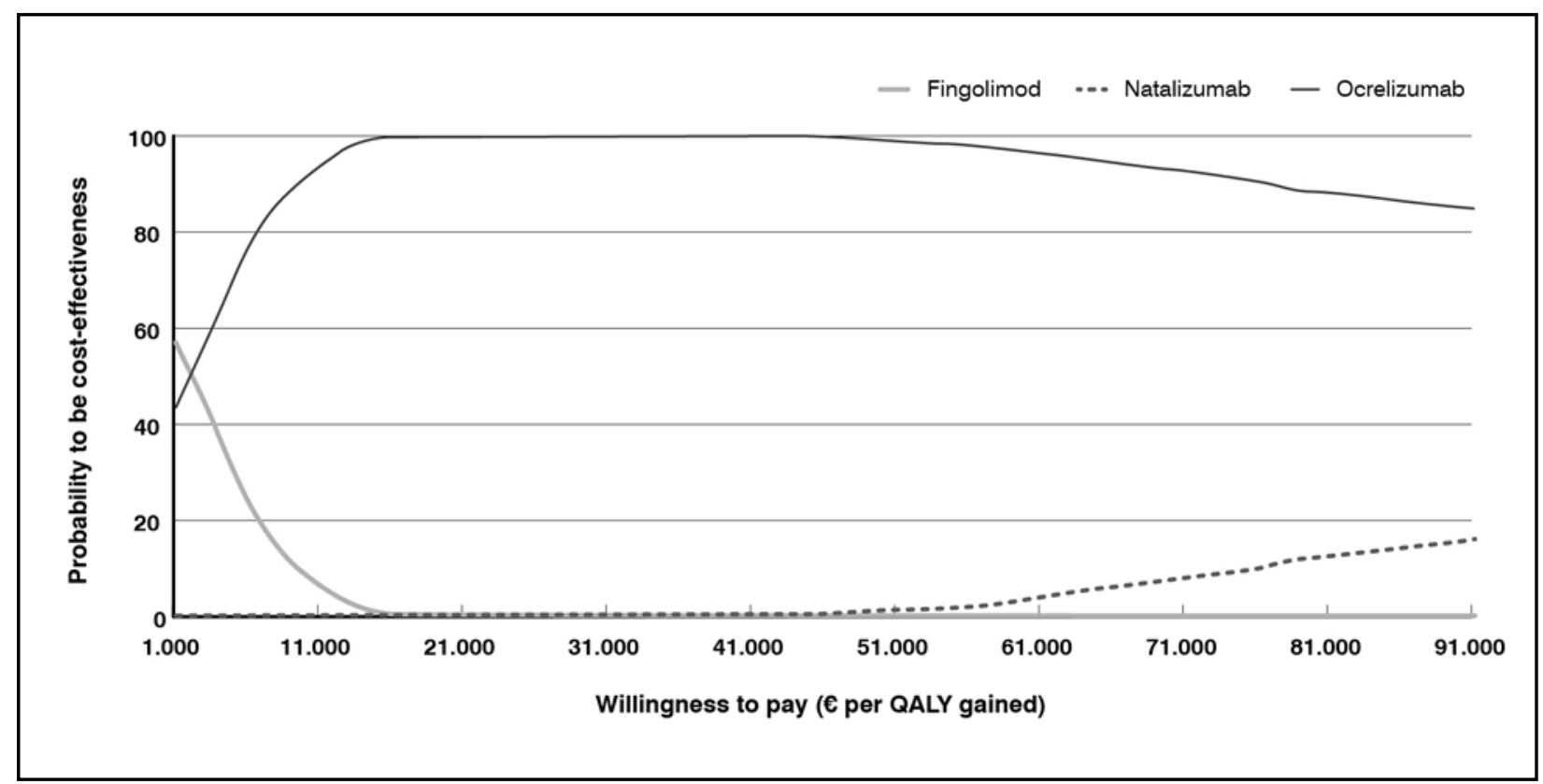

Figure 2. Cost-effectiveness acceptability curves

the epidemiological and market share data to estimate the 3 years overall cost of the Scenario with and without ocrelizumab. The difference between these two scenarios was estimated to assess the budget impact of ocrelizumab in Italy over a 3-year time horizon.

\section{RESULTS}

\section{Cost-Effectiveness Analysis}

In the base case scenario, ocrelizumab resulted as the most effective treatment with 0.504 QALYs gained compare to natalizumab and 1.306 QALYs compare to fingolimod (Table VI). Further, ocrelizumab was less expensive than natalizumab and with an incremental cost of $€$ 2,645 compared to fingolimod. Comparing ocrelizumab with fingolimod, the ICER estimated was $€ 2,023$ per QALY gained, while ocrelizumab resulted cost-saving (less expensive and more effective) compared to natalizumab.

In the alternative scenario 1, using the CDP-24 weeks as effectiveness outcome, ocrelizumab resulted cost-saving compared to fingolimod and cost-effective compared to natalizumab (Table VI). Further, using the society perspective, that includes the high non-medical and -indirect costs associated to MS (Alternative scenario 2) [21], ocrelizumab resulted less expensive and more effective, when compared to both fingolimod and natalizumab (Table VI).

The results of the one-way sensitivity analysis reported that ocrelizumab is always the cost-effective option compared to ocrelizumab and fingolimod. These results were confirmed by the probabilistic sensitivity analysis (Figure 2) where ocrelizumab reported a $99 \%$ probability to be cost-effectiveness at a willingness to pay threshold of $€ 40,000$ per QALY gained.

\section{Budget impact analysis}

Our model estimated a target population of RMS equal to 14,966 patients in the first year, 15,462 in the second year and 15,955 in the third year. The number of patients treated with each DMT in the two scenarios is reported in Supplementary Files, Figure 1. Fingolimod reported the higher number of patients treated in the Scenario No-Ocre $(8,980$ first year, 8,277 second year, and 9,573 third year); while in the scenario with ocrelizumab, fingolimod was the most used therapy only in the first and second year and ocrelizumab in the third year. In the base case analysis, the total economic impact of ocrelizumab patients with RMS was estimated at $€ 21$ million in the 3 years simulated, with an overall budget reduction of $2.6 \%$ (Figure 3). The model reported a budget reduction due to ocrelizumab of $1.01 \%$ ( $-€ 2.64$ millions) in the first year, $2.51 \%$ (-€ 6.77 millions) in the second year, and $4.20 \%$ ( $-€ 11.69$ millions) in the third year. 


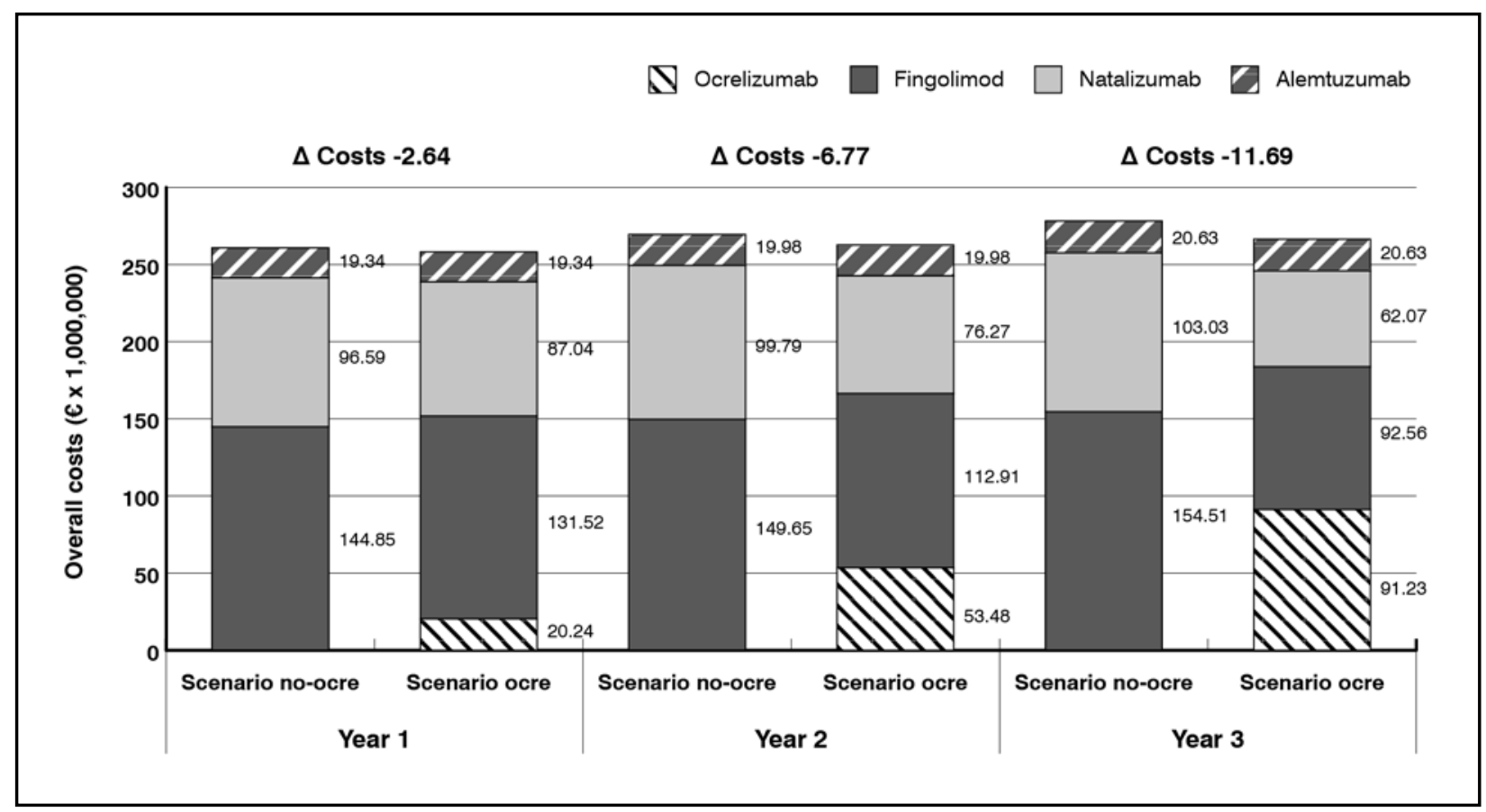

Figure 3. Budget estimated for each treatment in the two scenario and budget impact estimation for each simulated year

\section{DISCUSSION}

Ocrelizumab is a humanized anti-CD20 monoclonal antibody recently approved in Italy for the treatment of adults with RMS and PPMS. In our study we compared this new treatment option with the most used second-line DMTs in Italy, in order to estimate ocrelizumab cost-effectiveness in RMS patients. Further, to provide a complete picture of the economic impact of this new DMT, we assessed the possible budget impact associated to its use in the Italian market.

In two analyses conducted, ocrelizumab resulted cost-effective compared to natalizumab and fingolimod with a positive impact on the overall Italian NHS budget. In the base-case analysis the ICER per QALY gained was $€ 2,023$ for ocrelizumab compared to fingolimod, while ocrelizumab resulted cost-saving compared to natalizumab. The sensitivity analysis confirmed the results. Further, the use of ocrelizumab in Italy was associated to a budget decrease of $€ 21$ million in a 3-year time horizon, with an overall budget reduction of $2.6 \%$.

To our knowledge, our analyses are the first conducted to determinate the cost-effectiveness and budget impact of ocrelizumab in Italy. Our cost-effectiveness results are in line with previous analyses conducted in other countries [38-40]. The Scottish medicines Consortium (SMC) reported ocrelizumab as the cost-effectiveness option compare to natalizumab and fingolimod [38]. Using the CDP-12 and CDP-24 as treatment efficacy outcomes, ocrelizumab resulted cost-saving and cost-effective compared to natalizumab and reported an ICER between $£ 1,400$ and $£ 4,000$ per QALY gained compare to fingolimod. In a cost-effectiveness study conducted in US, ocrelizumab resulted more effective (8.48 QALYs) and less costly $(\$ 908,365)$ compared to both natalizumab $(8.46$ QALYs and $\$ 1,048,599)$ and fingolimod (7.96 QALYs and \$1,085,814) [40]. Similar results were reported in the analysis conducted by the Canadian Agency for Drugs and Technologies in Health [39], in which ocrelizumab reported a lower cost $(\$ 1,001,296)$ and higher efficacy $(12.79$ QALYs) compared to fingoli$\bmod (\$ 1,007,689$ and 12.24 QALYs), and a lower cost with slightly lower efficacy compared to natalizumab $(\$ 1,127,130$ and 12.99 QALYs) that makes ocrelizumab the cost-effective treatment option.

No data are published about the budget impact associated to ocrelizumab in Italy or other countries. Our study is the first providing information on ocrelizumab budget impact, showing a possible reduction in RMS costs of $€ 21$ million (2.6\%) in a 3-year time horizon, giving also the alternative possibility to treat more MS patients at the same overall budget impact. As showed in our analysis, the reduction of the budget was associated to the use of ocrelizumab instead of fingolimod and natalizumab. These results look in line with the cost-effectiveness analyses performed in other countries, where ocrelizumab frequently resulted less expensive 
than natalizumab and fingolimod [38-40]. However, new budget impact analyses are required to confirm these results in other countries.

Among limitations of this study, some reside on the data source for treatments efficacy. The model based the treatment efficacy (relapse rate and disease progression) on a NMA conducted on the DMTs clinical trials [24]. Further, also the probability of treatment interruption for any causes was based on the same NMA. Clinical trials included in the NMA had usually a short-term follow-up (maximum 2 years) compare to the disease duration and the simulation time-horizon. These data need to be updated with longer follow-up to understand the possible relative efficacy of the treatment included. However, using indirect comparison analysis is the recommended approach in the guidelines to manage missing direct comparison between treatments [41]. Another limitation is associated to the exclusion of alemtuzumab within the treatments. Alemtuzumab presents some different characteristics compared to the other second line DMTs that make difficult its inclusion in our evaluation. All second-line DMTs need to be administered chronically until the lack of efficacy or safety issue development, while alemtuzumab is provided initially as 2 annual treatment courses and in case of clinical or magnetic resonance imaging disease activity retreatment is necessary after the initial 2 annual treatment courses [42]. Based on the available data, patients treated with alemtuzumab reported retreatment in $36.1 \%$ in years 3,4 , or $5(25.6 \%$ received 1 additional course, $9.0 \%$ received 2 additional courses, and $1.5 \%$ received 3 additional courses) [43]. While over 10-year time horizon, $43 \%, 12 \%$, and $10 \%$ received three, four, or five courses of alemtuzumab, respectively [44]. These data make some issues in modeling efficacy and costs over a lifetime time horizon, particularly considering the simulation of treatment effect over time, treatment discontinuation and efficacy of retreatment [45]. Based on our model structure and long-term treatment effect and discontinuation, we decided to not include alemtuzumab in this analysis and focused on fingolimod and natalizumab that represented the $95 \%$ of the second-line DMTs used in Italy, and are chronic treatment as ocrelizumab. Based on this approach we include alemtuzumab only in the budget impact analysis but we assumed no change in its market share due to the market access of ocrelizumab.

\section{CONCLUSIONS}

The results of our cost-effectiveness and budget impact models reported ocrelizumab as an effective, efficacy, and efficient treatment in patients with RMS, who failed a first line DMT. Further, ocrelizumab showed the possibility to have a positive impact on the Italian NHS budget with a cost-saving of $€ 21$ million over a 3-year time horizon. The results of the model need to be confirmed by further economic evaluations based on ocrelizumab long-term data and including all DMTs.

\section{Funding}

This study was supported by an unrestricted research grant provided by Roche S.p.A.

\section{Conflicts of interest}

P.A.C. reports grants from Shire and personal fees from Pfizer and Roche, outside the submitted work. D.P. reports personal fees from Biogen Idec, Merck-Serono, TEVA, Novartis, Sanofi-Genzyme, and Almirall, outside the submitted work.

M.C. reports personal fees from Biogen Idec, Merck-Serono, TEVA, Novartis, Sanofi-Genzyme, Almirall, and Roche, outside the submitted work.

P.C. has nothing to disclose.

L.G.M. reports grants from Roche, during the conduct of the study; grants from MSD and Janssen, personal fees from Bayer, outside the submitted work.

\section{REFERENCES}

1. GBD 2015 Neurological Disorders Collaborator Group. Global, regional, and national burden of neurological disorders during 1990-2015: a systematic analysis for the Global Burden of Disease Study 2015. Lancet Neurol 2017; 16: 877-97; https://doi.org/10.1016/ S1474-4422(17)30299-5

2. Brownlee WJ, Hardy TA, Fazekas F, et al. Diagnosis of multiple sclerosis: progress and challenges. Lancet 2017; 389: 1336-46; https://doi.org/10.1016/S0140-6736(16)30959-X 
3. Reich DS, Lucchinetti CF, Calabresi PA. Multiple Sclerosis. N Engl J Med 2018; 378 : 169-80; https://doi.org/10.1056/NEJMra1401483

4. Adelman G, Rane SG, Villa KF. The cost burden of multiple sclerosis in the United States: a systematic review of the literature. J Med Econ 2013; 16: 639-47; https://doi.org/10.311 $1 / 13696998.2013 .778268$

5. Kobelt G, Thompson A, Berg J, et al.; MSCOI Study Group; European Multiple Sclerosis Platform. New insights into the burden and costs of multiple sclerosis in Europe. Mult Scler 2017; 23: 1123-36; https://doi.org/10.1177/1352458517694432

6. Kobelt G, Berg J, Lindgren P, et al. Costs and quality of life of patients with multiple sclerosis in Europe. J Neurol Neurosurg Psychiatry 2006; 77: 918-26; https://doi.org/10.1136/ jnnp.2006.090365

7. Capra R, Cordioli C, Rasia S, et al. Assessing long-term prognosis improvement as a consequence of treatment pattern changes in MS. Mult Scler 2017; 23: 1757-61; https://doi. org/10.1177/1352458516687402

8. Hauser SL, Bar-Or A, Comi G, et al.; OPERA I and OPERA II Clinical Investigators. Ocrelizumab versus Interferon Beta-1a in Relapsing Multiple Sclerosis. N Engl J Med 2017; 376: 221-34; https://doi.org/10.1056/NEJMoa1601277

9. Iannazzo S, Iliza AC, Perrault L. Disease-Modifying Therapies for Multiple Sclerosis: A Systematic Literature Review of Cost-Effectiveness Studies. Pharmacoeconomics 2018; 36: 189-204; https://doi.org/10.1007/s40273-017-0577-2

10. Sanders GD, Neumann PJ, Basu A, et al. Recommendations for Conduct, Methodological Practices, and Reporting of Cost-effectiveness Analyses: Second Panel on Cost-Effectiveness in Health and Medicine. JAMA 2016; 316: 1093-103; https://doi.org/10.1001/ jama.2016.12195

11. Cortesi PA, Belli LS, Facchetti R, et al.; European Liver and Intestine Transplant Association (ELITA). The optimal timing of hepatitis $\mathrm{C}$ therapy in liver transplant-eligible patients: Cost-effectiveness analysis of new opportunities. J Viral Hepat 2018; 25: 791-801; https:// doi.org/10.1111/jvh.12877

12. Cottrell DA, Kremenchutzky M, Rice GP, et al. The natural history of multiple sclerosis: a geographically based study. 5. The clinical features and natural history of primary progressive multiple sclerosis. Brain 1999; 122: 625-39; https://doi.org/10.1093/brain/122.4.641

13. Kremenchutzky M, Rice GP, Baskerville J, et al. The natural history of multiple sclerosis: a geographically based study 9: observations on the progressive phase of the disease. Brain 2006; 129: 584-94; https://doi.org/10.1093/brain/awh721

14. Scalfari A, Neuhaus A, Degenhardt A, et al. The natural history of multiple sclerosis, a geographically based study 10: relapses and long-term disability. Brain 2010; 133: 191429; https://doi.org/10.1093/brain/awq118

15. Patzold U, Pocklington PR. Course of multiple sclerosis. First results of a prospective study carried out of 102 MS patients from 1976-1980. Acta Neurol Scand 1982; 65: 248-66; https://doi.org/10.1111/j.1600-0404.1982.tb03084.x

16. Weinshenker BG, Bass B, Rice GP, et al. The natural history of multiple sclerosis: a geographically based study. 2. Predictive value of the early clinical course. Brain 1989; 112: 1419-28; https://doi.org/10.1093/brain/112.6.1419

17. Orme M, Kerrigan J, Tyas D, et al. The effect of disease, functional status, and relapses on the utility of people with multiple sclerosis in the UK. Value Health 2007; 10: 54-60; https:// doi.org/10.1111/j.1524-4733.2006.00144.x

18. National Institute For Health And Clinical Excellence- NICE. Fingolimod for the Treatment of Relapsing-Remitting Multiple Sclerosis in Adults. Revised in line with ERG responses, and updated indication, in line with CHMP opinion: Submitted 18 March 2011. Available at https://www.nice.org.uk/guidance/TA254/documents/manufacturer-submission-fromnovartis2 (last accessed November 2018)

19. Pokorski RJ. Long-term survival experience of patients with multiple sclerosis. J Insur Med 1997; 29: 101-6 
20. Palace J1, Bregenzer T, Tremlett H, Oger J, Zhu F, Boggild M, Duddy M, Dobson C. UK multiple sclerosis risk-sharing scheme: a new natural history dataset and an improved Markov model. BMJ Open 2014; 4: e004073

21. Battaglia M, Kobelt G, Ponzio M, et al.; European Multiple Sclerosis Platform. New insights into the burden and costs of multiple sclerosis in Europe: Results for Italy. Mult Scler 2017; 23: 104-16; https://doi.org/10.1177/1352458517708176

22. Cozzolino P, Cortesi PA, Cesana G, et al. The Economic Burden of Different Multiple Sclerosis Phenotypes. Value in Health 2017; 20: A721; https://doi.org/10.1016/j. jval.2017.08.1934. Poster available at: https://tools.ispor.org/RESEARCH_STUDY_DIGEST/research_index.asp (last accessed July 2019)

23. Istituto Nazionale di Statistica - ISTAT. Mortality Tables 2015. Available at http://demo. istat.it/index.html (last accessed November 2018)

24. McCool R, Wilson K, Arber M, et al. Systematic review and network meta-analysis comparing ocrelizumab with other treatments for relapsing multiple sclerosis. Mult Scler Relat Disord 2019; 29: 55-61; https://doi.org/10.1016/j.msard.2018.12.040

25. Biogen Idec Ltd, Heron Evidence Development. Natalizumab (Tysabri ${ }^{\circledR}$ ) for the Treatment of Adults with Highly Active Relapsing Remitting Multiple Sclerosis: Biogen Idec Single Technology Appraisal (STA) Submission to the National Institute for Health and Clinical Excellence. London: NICE; 2007. URL: www.nice.org.uk/guidance/TA127/documents/ multiple-sclerosis-natalizumab-manufacturer-submissions-biogen-idec-uk-and-elanpharma-international-ltd-joint-development-agreement-confidential-information-removed2 (last accessed November 2018)

26. National Institute For Health And Clinical Excellence - NICE. Alemtuzumab for treating relapsing-remitting multiple sclerosis. Technology appraisal guidance TA312. London: NICE, 2014. Available at www.nice.org.uk/guidance/ta312 (last accessed November 2018)

27. Istituto Nazionale di Statistica - ISTAT. Calcolo delle rivalutazioni monetarie. Available at http://rivaluta.istat.it/Rivaluta/ (last accessed November 2018)

28. Agenzia Italiana del Farmaco - AIFA. Tabelle farmaci di classe A e H al 17/12/2018. Available at http://www.aifa.gov.it/content/tabelle-farmaci-di-classe-e-h-al-17122018 (last accessed December 2018)

29. AGEnzia NAzionale per i Servizi Sanitari Regionali-AGENAS. Prestazioni specialistiche ambulatoriali. Confronto tra le tariffe nazionali ex DM 18.10.2012 e le tariffe regionali vigenti al 31.10.2014. Available at http://www.agenas.it/images/agenas/monitoraggio/ spesa_sanitaria/tariffe/specialistica_ambulatoriale_tariffe.pdf (last accessed November 2018)

30. Regione Emilia-Romagna. Assessorato Politiche per la Salute. Percorso regionale di diagnosi e terapia della sclerosi multipla. Maggio 2011. Available at http://www.saluter.it/ documentazione/ptr/elaborati/128_sclerosi_multipla.pdf (last accessed November 2018)

31. Regione Emilia-Romagna. Assessorato Politiche per la Salute. Indicazioni per erogazione del fingolimod presso i centri sclerosi multipla dell'Emilia Romagna. Maggio 2012. Available at http://salute.regione.emilia-romagna.it/documentazione/ptr/elaborati/159_fingolimod.pdf/at_download/file/159_fingolimod.pdf (last accessed November 2018)

32. Associazione Italiana Sclerosi Multipla - AISM. Barometro della sclerosi multipla 2018

33. Atlas of MS 2013: Mapping Multiple Sclerosis Around the World. London: Multiple Sclerosis International Federation; 2013. Available at: http:/www.msif.org/about-ms/publications-and-resources/ (last accessed November 2018)

34. Jick SS, Li L, Falcone GJ, et al. Mortality of patients with multiple sclerosis: a cohort study in UK primary care. J Neurol 2014; 261: 1508-17, https://doi.org/10.1007/s00415014-7370-3

35. Istituto Nazionale di Statistica - ISTAT. Popolazione Residente per età, sesso e stato civile. Available at http://demo.istat.it/ (last accessed November 2018) 
36. Cortesi PA, Cozzolino P, Cesana G, et al. The Prevalence and Treatment Status of Different Multiple Sclerosis Phenotypes in a Italian Reference Center. Value in Health 2017; 20: A720; https://doi.org/10.1016/j.jval.2017.08.1928

37. Ciaccio A, Cortesi PA, Bellelli G, et al. Direct-acting antivirals combination for elderly patients with chronic hepatitis C: A cost-effectiveness analysis. Liver Int 2017; 37: 982-94; https://doi.org/10.1111/liv.13339

38. Scottish Medicine Consortium - SMC. Ocrelizumab $300 \mathrm{mg}$ concentrate for solution for infusion (Ocrevus ${ }^{\circledR}$ ). Advice, November 2018. Available at https://www.scottishmedicines. org.uk/medicines-advice/ocrelizumab-ocrevus-resubmission-smc2121/ (last accessed February 2019)

39. Canadian Agency for Drugs and Technologies in Health - CADTH. Pharmacoeconomic Review Report Ocrelizumab (OCREVUS). December 2017. Available at https://www.cadth. ca/ocrelizumab (last accessed February 2019)

40. Chirikov V, Ma I, Joshi N, et al. Cost-Effectiveness of Alemtuzumab in the Treatment of Relapsing Forms of Multiple Sclerosis in the United States. Value Health 2019; 22: 168-76; https://doi.org/10.1016/j.jval.2018.08.011

41. Hoaglin DC, Hawkins N, Jansen JP, et al. Conducting Indirect-Treatment-Comparison and Network-Meta-Analysis Studies: Report of the ISPOR Task Force on Indirect Treatment Comparisons Good Research Practices-Part 2. Value Health 2011; 14: 429-37; https://doi. org/10.1016/j.jval.2011.01.011

42. Fox EJ, Arnold DL, Cohen JA, et al. Durable efficacy of alemtuzumab on clinical outcomes over 5 years in CARE-MS II with most patients free from treatment for 4 years. Poster presented at ECTRIMS 2015.Barcelona, Spain, October 7-10, 2015

43. Havrdova E, Arnold DL, Cohen JA, et al.; CARE-MS I and CAMMS03409 Investigators. Alemtuzumab CARE-MS I 5-year follow-up: Durable efficacy in the absence of continuous MS therapy. Neurology 2017; 89: 1107-16; https://doi.org/10.1212/WNL.0000000000004313

44. Coles AJ, Habek M, Bass AD, et al. Durable efficacy of alemtuzumab over 10 years: longterm follow-up of patients with RRMS from the CAMMS223 Study. Neurology 2016; 78: P3.053

45. Tappenden P, McCabe C, Chilcott J, et al. Cost-effectiveness of diseasemodifying therapies in the management of multiple sclerosis for the Medicare population. Value Health 2009; 12: 657e65; https://doi.org/10.1111/j.1524-4733.2008.00485.x 